\title{
Additive Manufacturing Fabrication of PEEK Counter-Current Centrifugal Contactor Components
}

Chemical \& Fuel Cycle Technologies Division 


\begin{abstract}
About Argonne National Laboratory
Argonne is a U.S. Department of Energy laboratory managed by UChicago Argonne, LLC under contract DE-AC02-06CH11357. The Laboratory's main facility is outside Chicago, at 9700 South Cass Avenue, Lemont, Illinois 60439. For information about Argonne and its pioneering science and technology programs, see www.anl.gov.
\end{abstract}

\title{
DOCUMENT AVAILABILITY
}

Online Access: U.S. Department of Energy (DOE) reports produced after 1991 and a growing number of pre-1991 documents are available free at OSTI.GOV (http://www.osti.gov/),

a service of the US Dept. of Energy's Office of Scientific and Technical Information.

Reports not in digital format may be purchased by the public from the National Technical Information Service (NTIS):

U.S. Department of Commerce

National Technical Information Service

5301 Shawnee Road

Alexandria, VA 22312

www.ntis.gov

Phone: (800) 553-NTIS (6847) or (703) 605-6000

Fax: (703) 605-6900

Email: orders@ntis.gov

Reports not in digital format are available to DOE and DOE contractors from the Office of Scientific and Technical Information (OSTI):

U.S. Department of Energy

Office of Scientific and Technical Information

P.O. Box 62

Oak Ridge, TN 37831-0062

www.osti.gov

Phone: (865) 576-8401

Fax: (865) 576-5728

Email: reports@osti.gov

\section{Disclaimer}

This report was prepared as an account of work sponsored by an agency of the United States Government. Neither the United States Government nor any agency thereof, nor UChicago Argonne, LLC, nor any of their employees or officers, makes any warranty, express or implied, or assumes any legal liability or responsibility for the accuracy, completeness, or usefulness of any information, apparatus, product, or process disclosed, or represents that its use would not infringe privately owned rights. Reference herein to any specific commercial product, process, or service by trade name, trademark, manufacturer, or otherwise, does not necessarily constitute or imply its endorsement, recommendation, or favoring by the United States Government or any agency thereof. The views and opinions of document authors expressed herein do not necessarily state or reflect those of the United States Government or any agency thereof, Argonne National Laboratory, or UChicago Argonne, LLC. 
ANL/CFCT-19/22

\section{Additive Manufacturing Fabrication of PEEK Counter-Current Centrifugal Contactor Components}

by

Peter Kozak, Brian Saboriendo, Peter Tkac

Chemical \& Fuel Cycle Technologies Division, Argonne National Laboratory

October 23, 2019 



\section{CONTENTS}

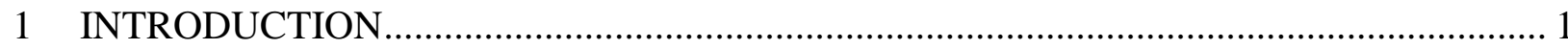

2 MACHINED PEEK CONTACTOR TESTING …...................................................... 1

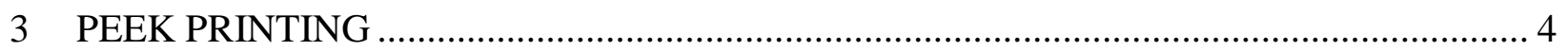

4 HYDRODYNAMIC TESTING OF PEEK PRINTED CCS STAGES ............................. 7

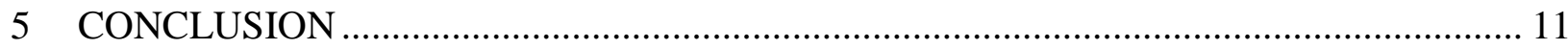

\section{FIGURES}

1 MOEX flowsheet utilized for testing of 10 stages of machined PEEK centrifugal

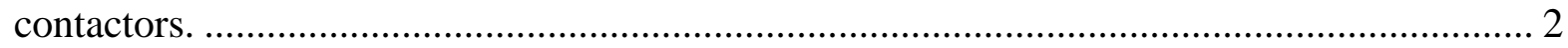

2 Bank of machined PEEK contactors with 10 stages. .................................................. 2

3 Concentration profiles of Mo and $\mathrm{K}$ in aqueous and organic phases in 10 stage setup with three feed stages, three scrub stages, and four strip stages. ..................................... 3

4 The images show PEEK filaments following extrusion, (left) before and (right) after

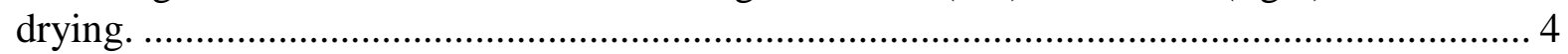

5 Semi-crystalline (left) vs amorphous (right) PEEK................................................... 5

6 PEEK printed 1.25-cm CCS housing used to demonstrate the material's leak-tightness......... 6

7 PEEK printed 3-cm CCS rotor used to determine hydrodynamic performance. ................... 6

8 PEEK printed 2-cm CCS rotor used for zero-point and holdup testing .............................. 8

9 3-cm stage used for zero-point and holdup testing. Note that the rotor is PEEK and the housing is inkjet-printed acrylic............................................................................. 8

10 Flow out from the light-phase weir as a percentage of the total flow rate [\%] vs feed rate $[\mathrm{mL} / \mathrm{min}]$. The zero-point is defined as the point where the line diverges significantly from zero. Blue $=$ acrylic standard rotor design; Orange $=$ acrylic improved rotor design; Purple = PEEK improved rotor design...................................... 9

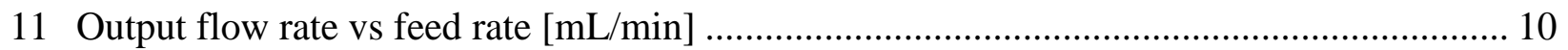

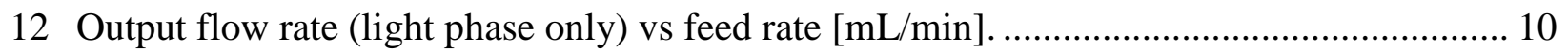

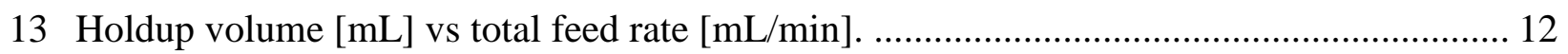




\section{FIGURES (Cont.)}

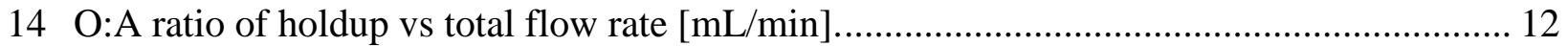

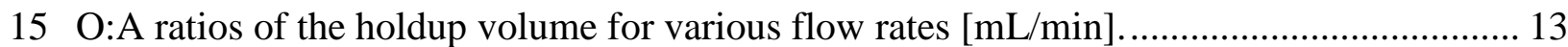




\section{INTRODUCTION}

Additive manufacturing (AM, also known as 3D printing) offers significant advantages for the fabrication of annular centrifugal contactor-separator (CCS) stages, particularly when complemented by post-processing via traditional machining. The shortcomings of 3D printing of CCSs are offset by its low cost, quick turnaround, and ability to manufacture enhanced mixing sections and multistage modules. High-density acrylic materials show promise for improved chemical resistance and mechanical properties. The demonstration of the MOEX process using acrylic AM CCS stages confirmed their full functionality and showed significant flexibility in geometry design while using relatively low-cost plastic materials. Over the past two years, AM has been used to produce low-cost centrifugal contactor components, ranging from bench-scale laboratory testing to a full-scale industrial process with on-hand availability of spare parts and great flexibility in design modifications and quick testing and implementation. However, the shortcomings of stereolithography assembly (SLA)-printed acrylic components became apparent rather quickly. By the completion of the initial MOEX demonstration using the 9-stage 2-cm CCS bank, nearly half of the rotors had begun to fail from pitting and cracking. These problems were most likely caused by swelling of the rotor due to liquid absorption, resulting in improper excess runout, mechanical abrasion, and chemical exposure of the roughened surface. Additional deterioration of the acrylic stages was later discovered when the interiors of the acrylic housings began to experience flaking and delamination as the plastic dried out several weeks after the tests. All of these issues caused severe limitations to the life span of the CCS stages, which are acceptable for R\&D but undesirable for commercial-scale production.

Because of these exhibited limitations of SLA-printed acrylic, work was done to identify 3D-printable polymers with improved chemical compatibility characteristics and mechanical robustness. The polymers that were investigated included inkjet-fabricated acrylic, polypropylene, and polyether-ether-ketone (PEEK). While all the tested materials demonstrated improved chemical resistance when compared with the SLA-printed acrylic, PEEK was determined to have the ideal combination of low moisture absorption, chemical resistance, and mechanical robustness. On the basis of this conclusion, a 10-stage 3-cm CCS bank was fabricated using traditional machining and was tested. The PEEK material was demonstrated to survive long-term exposure to hydrochloric acid $(\mathrm{HCl})$, tributyl phosphate (TBP), and tetrachloroethylene (TCE).

\section{MACHINED PEEK CONTACTOR TESTING}

Seven contactor runs using three extraction, three scrub, and four strip stages were performed to test the compatibility and robustness of machined PEEK contactors. The schematic of the MOEX flowsheet is shown in Figure 1, and a photograph of the actual setup is shown in Figure 2. 


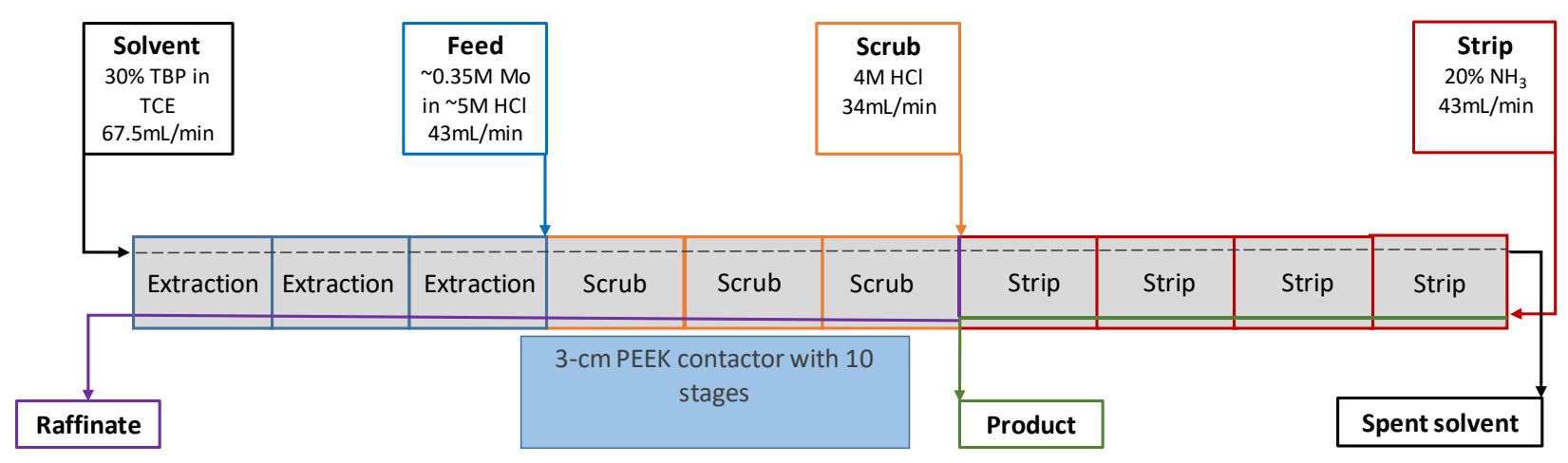

FIGURE 1 MOEX flowsheet utilized for testing of 10 stages of machined PEEK centrifugal contactors.

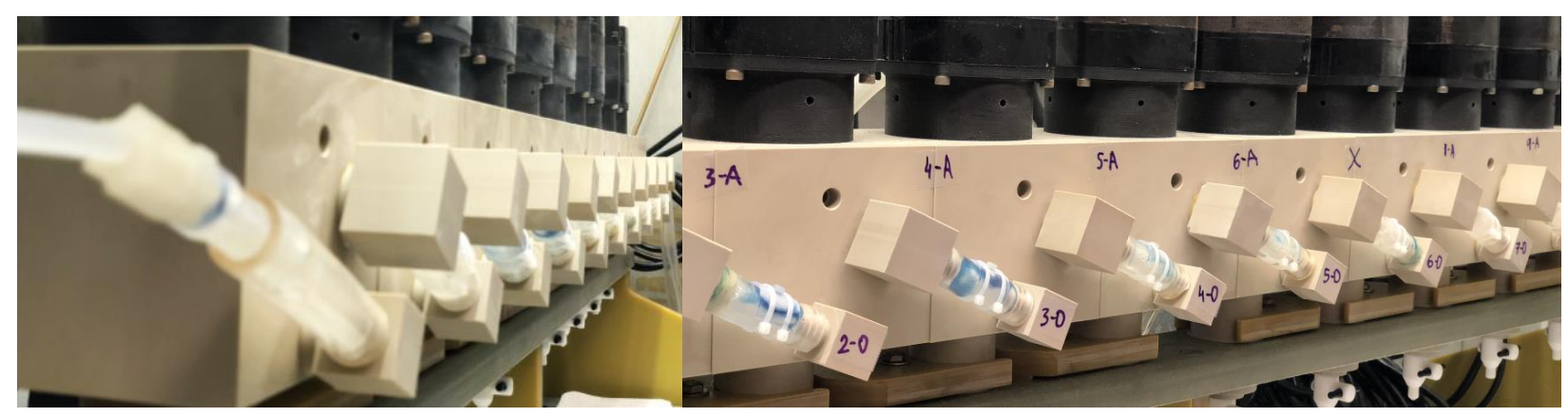

FIGURE 2 Bank of machined PEEK contactors with 10 stages.

After scaling up the rotors from $2 \mathrm{~cm}$ to $3 \mathrm{~cm}$, it was discovered that evaporation of TCE from the organic phase becomes more pronounced, as indicated by some phase carryover observed during the second run. The phase carryover was directly associated with a significant change in the density of the organic phase due to evaporation of TCE. As a corrective action, the density of the organic phase was monitored and adjusted before and after each run, to make sure the concentration of TBP remained at $30 \%$. It was discovered that during $\sim 2 \mathrm{hr}$ of running the $3-\mathrm{cm}$ bank, the density of TBP solution changed from $1.432 \mathrm{~g} / \mathrm{mL}$ to $1.419 \mathrm{~g} / \mathrm{mL}$. This change corresponds to a loss of $\sim 60 \mathrm{~mL}$ of TCE per liter of TBP/TCE, and a change in concentration of TBP from $30 \%$ to $32 \%$. It is therefore important to feed TCE into the organic phase reservoir during longer runs.

Typical concentration distributions of Mo and $\mathrm{K}$ in the organic and aqueous phases are shown in Figure 3. It was discovered that the concentration of potassium in the Mo product was slightly elevated compared to previous runs performed with AM 2-cm centrifugal contactors (1000-1600 ppm K versus 30-200 ppm K previously). This change was most likely due to the slightly different design of the traditionally manufactured PEEK contactor. Therefore, to decrease the concentration of $\mathrm{K}$ in the final strip, we reconfigured the 10-stage contactor process to four scrub stages and three strip stages, since complete recovery of Mo can be achieved with just three strip stages. Increasing the number of scrub stages ultimately decreased the concentration of $\mathrm{K}$ in the strip section to below the detection limit. 

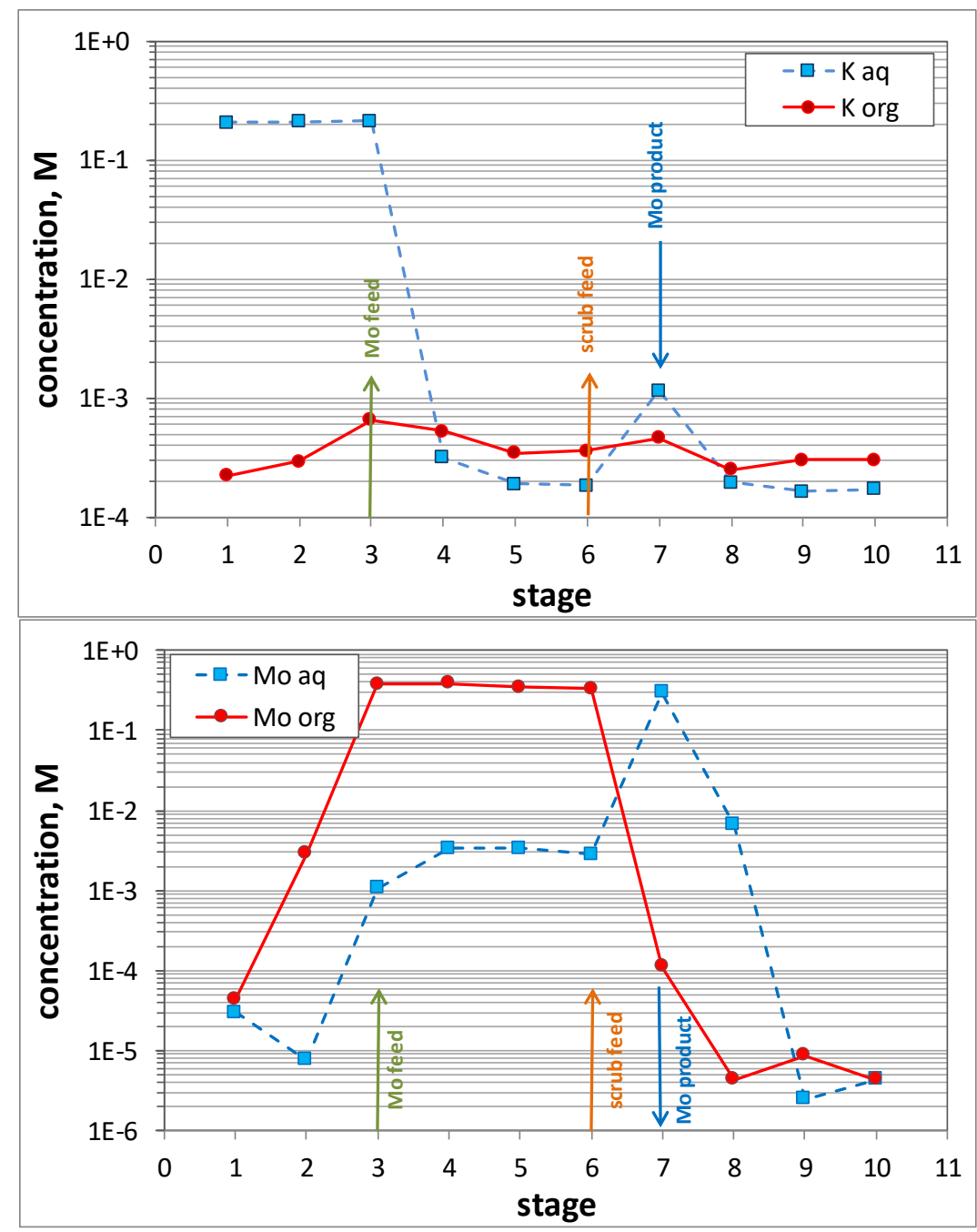

FIGURE 3 Concentration profiles of $M o$ and $K$ in aqueous and organic phases in 10 stage setup with three feed stages, three scrub stages, and four strip stages.

The PEEK contactor bank demonstrated some limitations as well, including the high cost of traditional manufacturing and chemical attack at weak points in components where PEEK parts were welded with epoxy. In addition, since the issue of chemical degradation of the contactor housing and rotors themselves had been mitigated, the life span of the motors became a new limiting factor as the metal bearings and exposed surfaces gradually corroded from exposure to $\mathrm{HCl}$ droplets and vapor.

Consequently, it was concluded that development of methods for 3D printing of contactor housings and rotors from PEEK was desirable. Having the capability to produce an AM contactor bank from PEEK would significantly increase the flexibility in design of centrifugal contactors. PEEK's advantages include (1) its excellent chemical resistivity in separation applications requiring corrosive reagents (such as $\mathrm{HCl}$ ) and chlorinated organic solvents (such as TCE, used in the MOEX process); (2) its formidable mechanical robustness; and (3) the ability to 
manufacture extended-residence-time stages that could improve the decontamination factor. Early attempts at printing with PEEK had identified interlayer adhesion and severe warping issues that needed to be resolved before these materials could be 3D-printed with the tolerances necessary for CCS stages.

\section{PEEK PRINTING}

Printing of PEEK was carried out via fused-filament fabrication (FFF), where a feedstock filament is extruded through a heated nozzle that is moved in order to trace out a single layer of material. When that layer is completed, the print head is either raised or the build platform is lowered so the next layer may be traced on top of the previous one. PEEK is a challenging material to fabricate using this technique because of its high melting temperature and semicrystalline microstructure, which results in significant volumetric contraction upon cooling, leading to warping and peeling during printing. This problem is mitigated by controlling the temperature of the entire build volume by printing in an enclosed, heated chamber. For this reason, an INTAMSYS FUNMAT Pro HT was procured, along with virgin PEEK (which was ultimately chosen for its better surface finish) and PEEK/Carbon Fiber filament from 3DXTech. Because of carbon fiber's low coefficient of thermal expansion, composite filament exhibits fewer warping issues at the cost of a higher surface roughness. During the humid summer months, environmental conditions were also found to cause problems with fabrication of the PEEK contactors. If the PEEK feedstock was left exposed to the surrounding environment (such as during long print jobs), it would absorb moisture. While PEEK does not readily absorb much moisture when compared with other plastics, the high nozzle temperature required to melt PEEK resulted in rapid expansion of water vapor absorbed by the filament as it extruded (see Figure 4), resulting in the formation of large bubbles and compromising the strength of the printed material. This issue was resolved by drying the filament in the FUNMAT's print chamber at $120^{\circ} \mathrm{C}$ for 12 hours and placing it in closed containers with desiccant packs during both storage and printing.

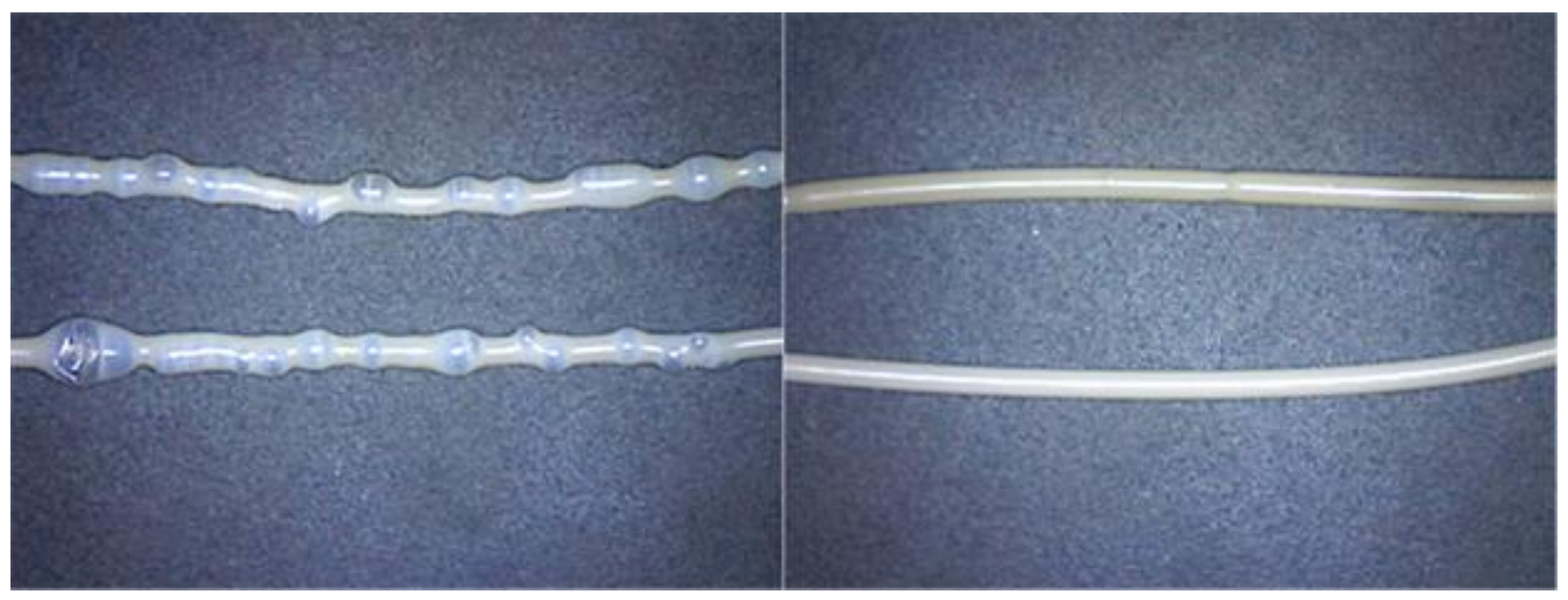

FIGURE 4 The images show PEEK filaments following extrusion, (left) before and (right) after drying. 
Largely through trial-and-error, the print settings were determined by starting with the manufacturer's specifications and then optimizing from there:

- Nozzle temperature $-410^{\circ} \mathrm{C}$

- Bed temperature $-155^{\circ} \mathrm{C}$

- Fan speed $-75 \%$

- Print speed $-50 \mathrm{~mm} / \mathrm{s}$

The majority of warping issues were eliminated by adjusting the build-bed/nozzle temperatures along with the fan setting, which controls how quickly the PEEK cools after being deposited. Ultimately, settings were determined at which the molten PEEK was cooled before it had a chance to crystallize, resulting in an amorphous microstructure (see Figure 5). This approach had three effects: (1) the amorphous polymer microstructure allows light to pass through unobstructed; (2) because there was no dramatic volume change as the PEEK crystallized, warping was minimized; and (3) the amorphous film was demonstrated to exhibit reduced brittleness and improved mechanical robustness.

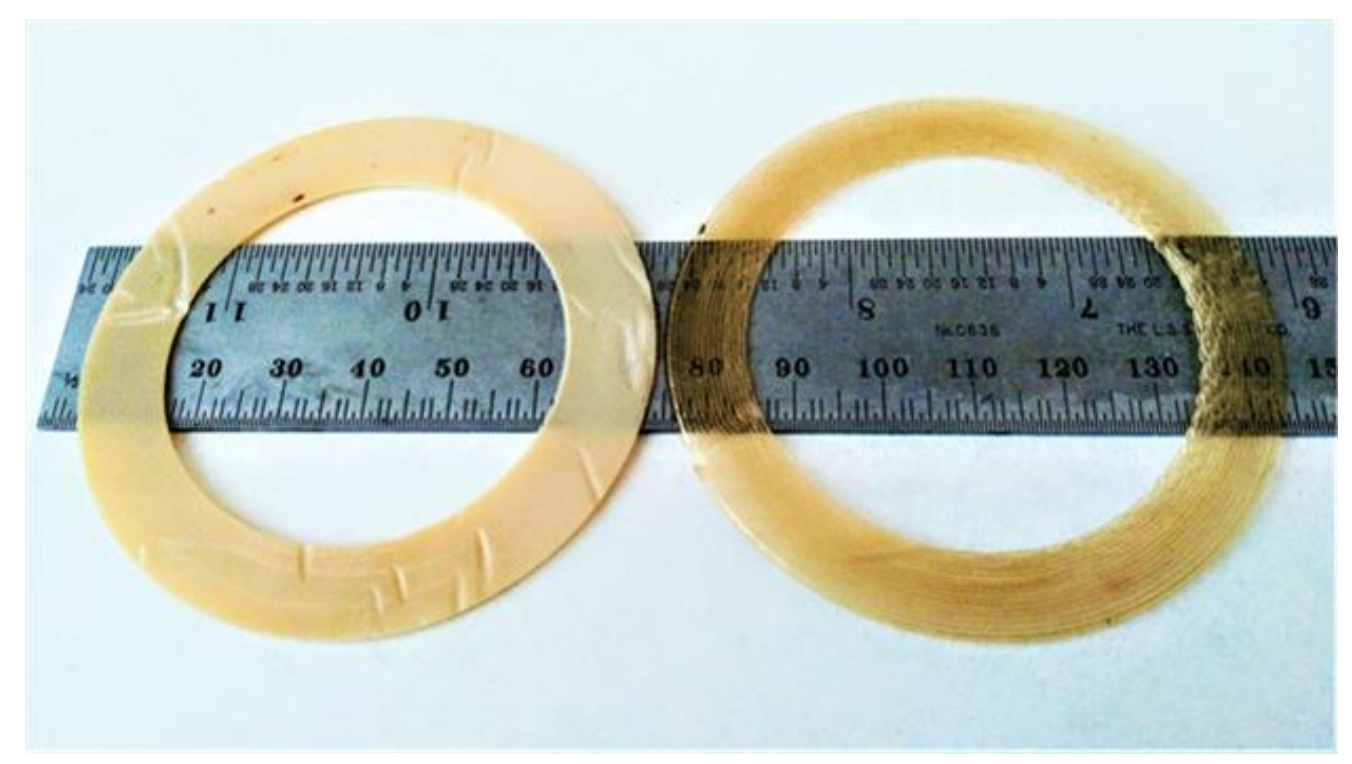

FIGURE 5 Semi-crystalline (left) vs amorphous (right) PEEK.

Bed-adhesion issues were also solved using a trial-and-error approach. While the manufacturer of the filament recommends Kapton tape, a more costly but more effective solution was to line the bed with an adhesive-backed PEEK film in order to minimize the chance of print failure due to peeling. The overall approach was as follows: Start with simple test prints that include geometric features shared with CCS housings and rotors, use PEEK to fabricate simple contactor components that are currently manufactured via traditional machining, and steadily increase the part complexity as more experience is gained in printing PEEK. Eventually, a number of full CCS components were printed from PEEK for testing, including a $1.25-\mathrm{cm}$ housing (see Figure 6) and 2-cm as well as 3-cm rotors (see Figure 7). 


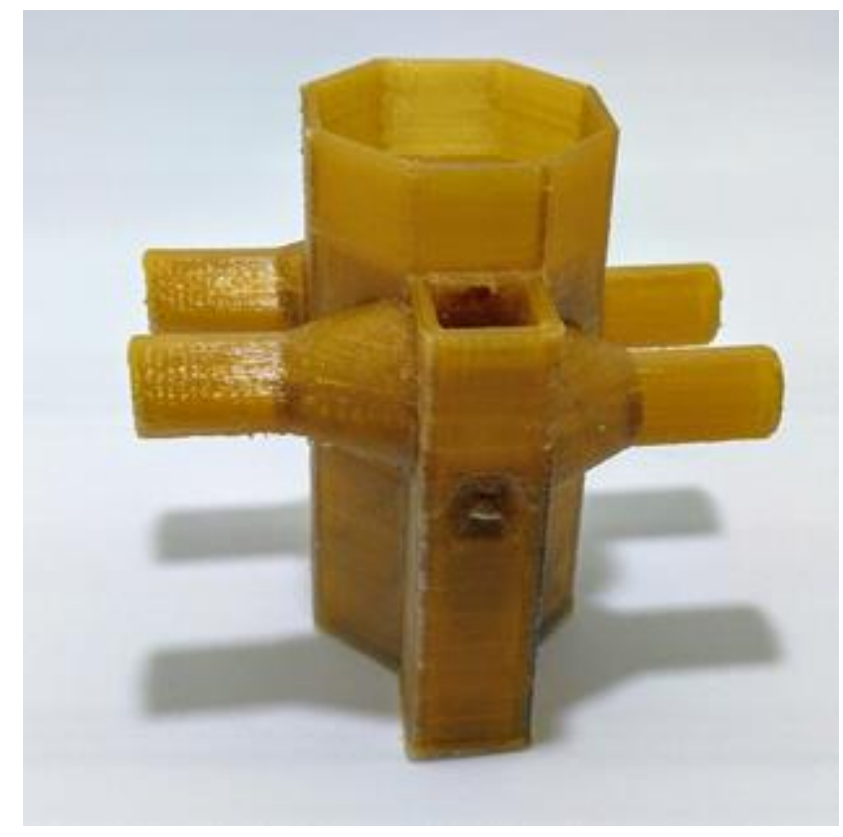

FIGURE 6 PEEK printed 1.25-cm CCS housing used to demonstrate the material's leak-tightness.

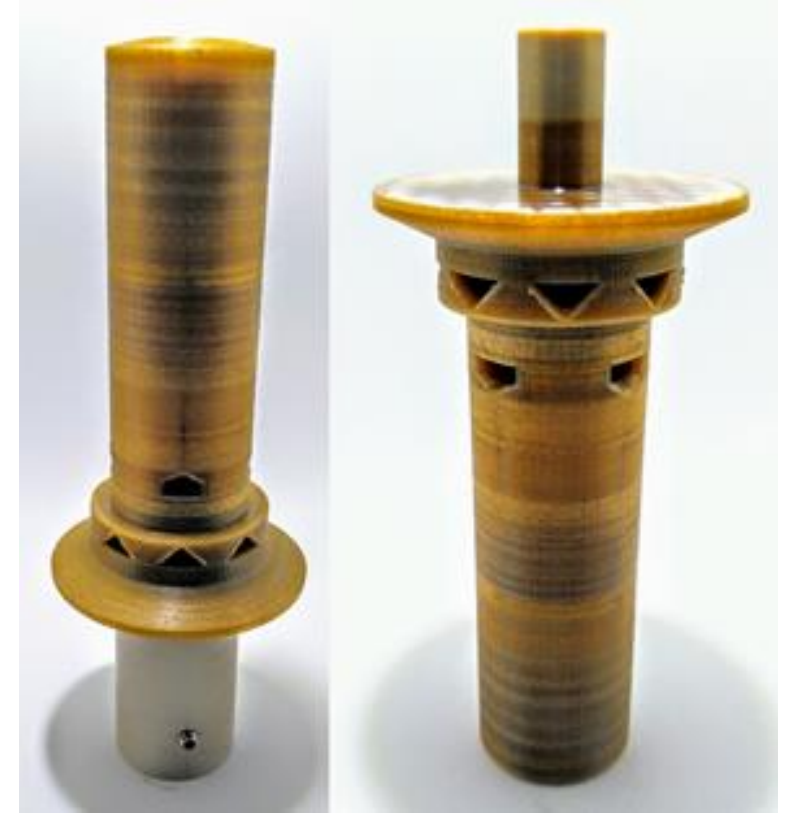

FIGURE 7 PEEK printed 3-cm CCS rotor used to determine hydrodynamic performance. 
While minimal post-processing was required for the housing, the rotors were turned in a lathe following printing in order to reduce runout and minimize surface roughness; this postprocessing was comparable to that required for other AM-fabricated rotors. The $1.25-\mathrm{cm}$ housing was tested (running nitric acid and dodecane) for several hours using an SLA-printed acrylic rotor and was found to hold solutions and perform similarly to other AM-fabricated housings. Owing to the simplicity of the housing's role in the operation of a CCS and the large amount of time/material required to print, efforts were focused on producing as many PEEK printed rotors as possible. As a result of this focus, along with mechanical problems with the FUNMAT printer late in the fiscal year, the $1.25-\mathrm{cm}$ housing was the only housing printed from PEEK.

\section{HYDRODYNAMIC TESTING OF PEEK PRINTED CCS STAGES}

In most cases, the rotor determines the hydrodynamic performance of a CCS stage. Exceptions include modification of the vanes at the bottom of the mixing section ${ }^{1}$ and implementation of an enhanced mixing section ${ }^{2}$, both of which are outside the scope of this work. Therefore, in order to test the effectiveness of PEEK printing for CCS fabrication, the performance of the rotors was measured via zero-point and holdup testing. Zero-point testing evaluates the rotor's phase-separation performance by determining the maximum throughput flow rate, i.e., the flow rate above which the heavy phase (in this case, water) breaks out and contaminates the light-phase weir; this flow rate is defined as the zero-point. Holdup testing is used to determine the contact time between the phases by measuring the volumes of phases present in the mixing and separating sections. Total holdup volume is measured by turning off the rotor and pump simultaneously and draining the solutions inside (nitric acid and dodecane) in order to measure the total volume and O:A ratio. The holdup contained within the mixing section is measured by simultaneously stopping the pumps and quickly draining the mixing section while the rotor is still spinning, which prevents the solutions contained within the rotor from being collected. The volume contained within the rotor can then be calculated as the difference between the total and mixing-section holdup volumes.

For simplicity, the 2-cm PEEK rotor (see Figure 8) was used for the majority of hydrodynamic testing, since the zero-point and holdups can be solved for as a function of the rotor diameter if the contactor geometry is similar. As a result, hydrodynamic testing of the $3-\mathrm{cm}$ rotor (see Figure 9) was limited to what was required to validate the $2-\mathrm{cm}$ rotor data. The $2-\mathrm{cm}$ PEEK rotor's performance was also compared with that of two SLA-printed 2-cm rotors, with one having the same design as the PEEK rotor and the other having the standard design used in past MOEX demonstrations. (The design updates were relatively minor and extended only to making the rotor design more compatible with FFF printing as well as streamlining the weir geometry.) This testing scheme allowed for two separate controls in order to determine whether

\footnotetext{
${ }^{1}$ Wardle, K. E., Allen, T. R., Anderson, M. H., \& Swaney, R. E. (2009). Analysis of the effect of mixing vane geometry on the flow in an annular centrifugal contactor. AIChE journal, 55(9), 2244-2259.

${ }^{2}$ Brown, M. A., Wardle, K. E., Lumetta, G., \& Gelis, A. V. (2016). Accomplishing equilibrium in ALSEP: demonstrations of modified process chemistry on 3-D printed enhanced annular centrifugal contactors. Procedia Chemistry, 21, 167-173.
} 
differences in performance between the rotors and historical data were due to the printing method or the rotor design.

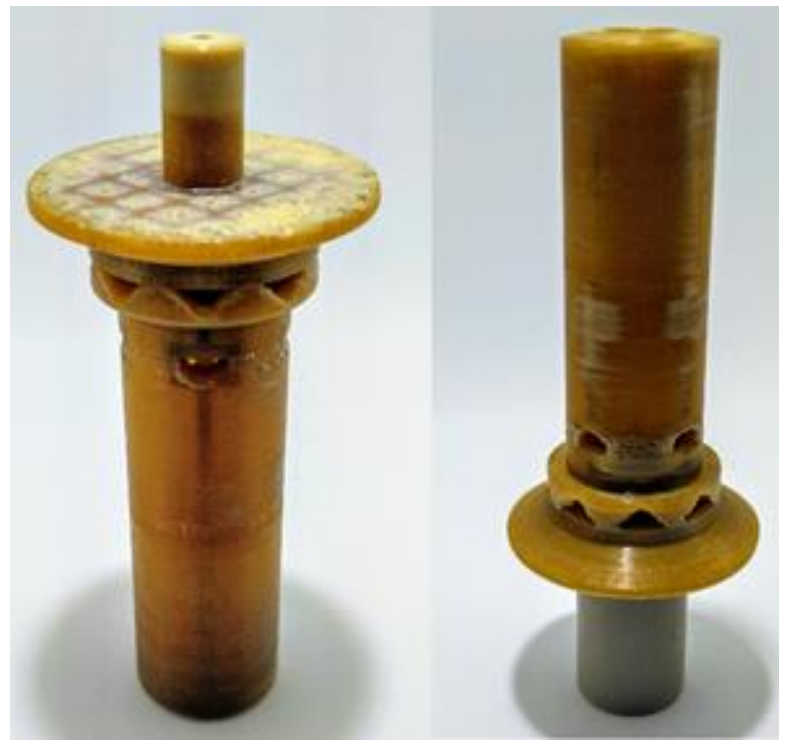

FIGURE 8 PEEK printed 2-cm CCS rotor used for zero-point and holdup testing.

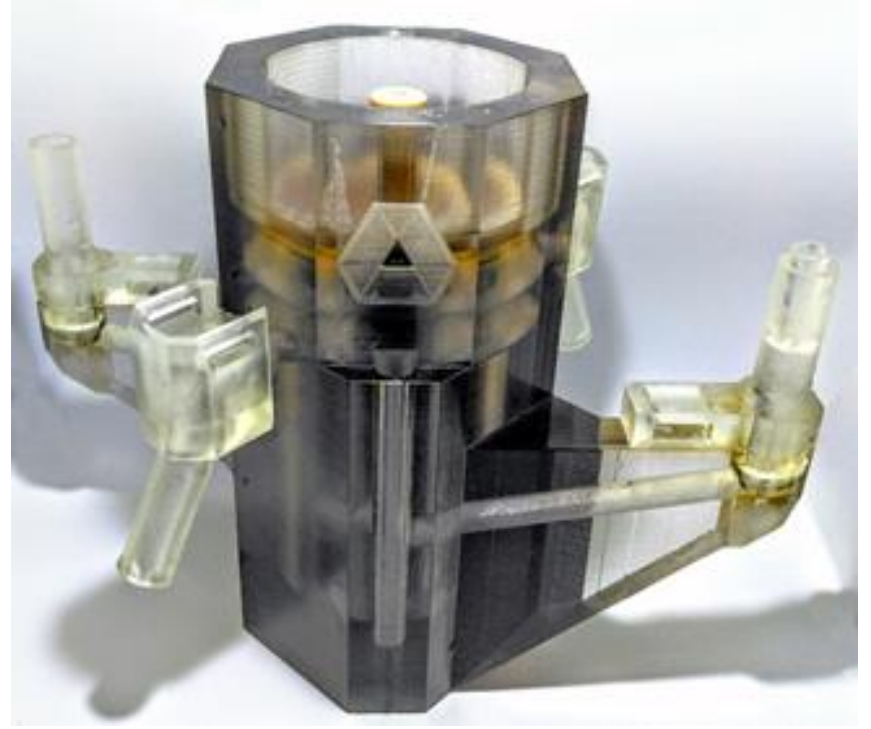

FIGURE 9 3-cm stage used for zero-point and holdup testing. Note that the rotor is PEEK and the housing is inkjet-printed acrylic. 
Results from the zero-point testing are shown in Figures 10, 11, and 12. The zero-point flow rate for the standard rotor agreed with historical data at approximately $60 \mathrm{~mL} / \mathrm{min}$, depending on acceptance criteria for an undetectable amount of breakthrough. The zero-point throughput for the SLA and PEEK improved rotor designs were consistent with one another at approximately $100 \mathrm{~mL} / \mathrm{min}$, representing a 67\% improvement upon the standard rotor design. These results suggest that the separation efficiency of the PEEK printed rotors is not significantly affected by surface roughness due to the FFF fabrication process, as had been considered. Furthermore, the fact that such a dramatic increase in performance was due to relatively minor streamlining of passages within the rotors strongly suggests that there are additional efficiencies to discover. Holdup testing was also carried out for the 3-cm improved PEEK rotor design, and the zero-point was determined to be $300 \mathrm{~mL} / \mathrm{min}$. This result can be used to confirm the cubic relationship between the rotor diameter and the zero-point, $q_{\max } \sim D^{3}$. On the basis of the zeropoint of $100 \mathrm{~mL} / \mathrm{min}$ for the 2-cm PEEK rotor, the predicted zero-point for the 3-cm rotor is $335 \mathrm{~mL} / \mathrm{min}$. This prediction validates the model and demonstrates that data may be extrapolated from one size rotor to another, assuming that the geometry and fabrication method are the same.

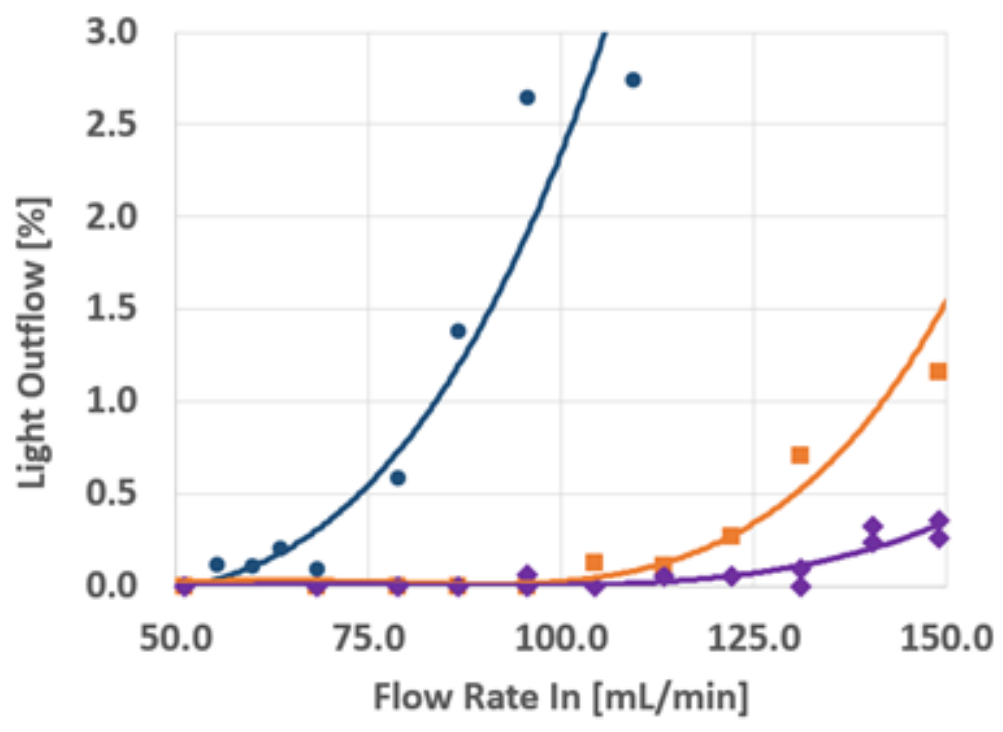

FIGURE 10 Flow out from the light-phase weir as a percentage of the total flow rate [\%] vs feed rate $[\mathrm{mL} / \mathrm{min}]$. The zero-point is defined as the point where the line diverges significantly from zero. Blue $=$ acrylic standard rotor design; Orange $=$ acrylic improved rotor design; Purple $=$ PEEK improved rotor design $^{3}$

\footnotetext{
${ }^{3}$ Candido Pereira, personal communication, October 10, 2019.
} 

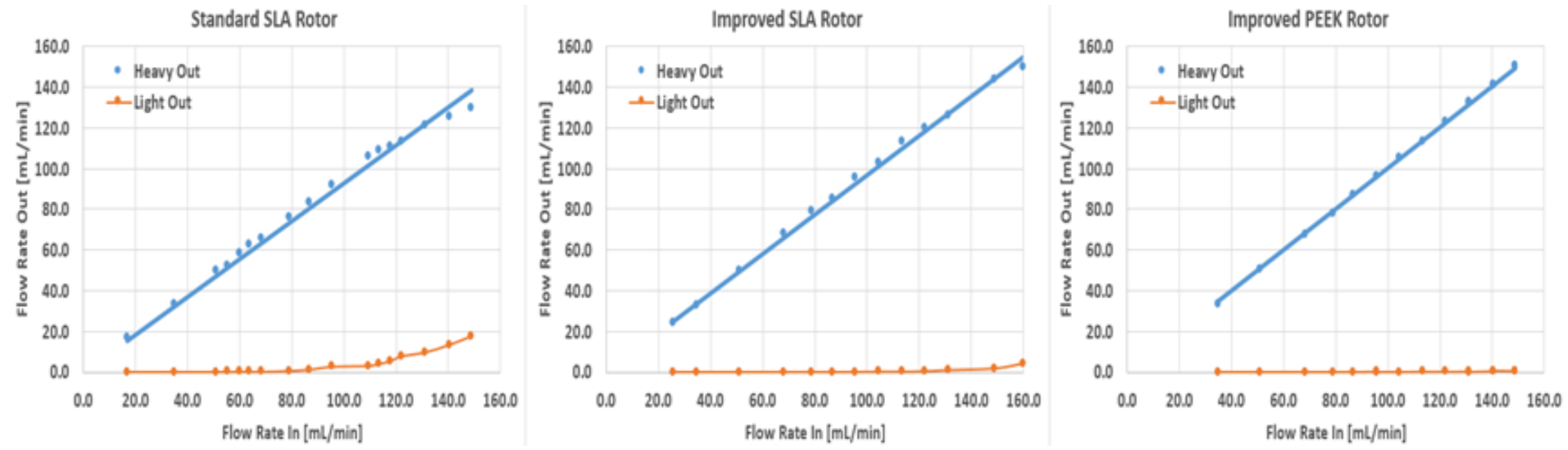

FIGURE 11 Heavy phase flow rate exiting the heavy phase (blue) and light phase (orange) outlets] vs feed flow rate [mL/min].
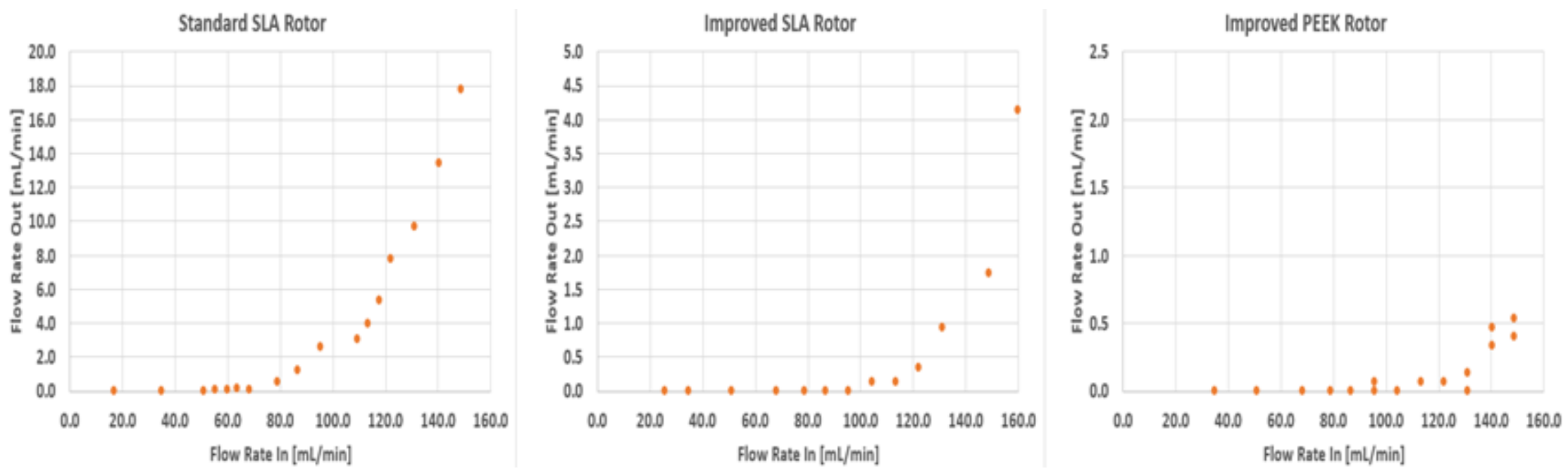

FIGURE 12 Heavy phase output flow rate exiting light phase outlet $[\mathrm{mL} / \mathrm{min}]$ vs feed flow rate $[\mathrm{mL} / \mathrm{min}] .^{4}$

${ }^{4}$ Candido Pereira, personal communication, October 10, 2019. 
Results from holdup testing are shown in Figures 13, 14, and 15. The holdup volumes for each case were consistent with one another when measured vs flow rate and input O:A ratio. However, the composition of the solutions contained within the mixing section varied greatly when the results for each rotor were compared. The light-phase concentration in the SLA-printed improved rotor design was roughly equivalent to the input O:A throughout a wide range of flow rates and concentrations. However, as shown in Figure 12, more light-phase solution seemed to be accumulating in the mixing section at high flow rates. It should be noted that this discrepancy is most dramatic at high flow rates, well above normal operating conditions. Additional tests will need to be carried out in which the flow rate is kept constant and the input O:A ratio is varied.

\section{CONCLUSION}

During FY 2019, a FFF printer with a temperature-controlled build volume was procured and techniques were developed to reliably print PEEK components. Several proof-of-concept rotors and housings of various sizes were fabricated and hydrodynamically tested. Hydrodynamic tests including leak-tightness and zero-point testing demonstrated comparable performance between PEEK printed and SLA-printed acrylic CCS stages of the improved design, and dramatically improved performance for PEEK printed stages when compared with the standard rotor design. With the AM fabrication of PEEK contactor stages and the implementation of a gas purge to protect the motor, all components are now either chemically resistant to attack by $\mathrm{HCl}$ and TCE or are protected from potential splashing and vapors. Future work will include carrying out additional holdup testing to better understand the composition of phases within the mixing section during typical operations. A 3-cm contactor housing will need to be printed from PEEK and integrated into an existing, traditionally-fabricated 3-cm PEEK CCS bank. This integration will further prove the performance of PEEK printed contactor stages and will allow for longer-term reliability testing. 

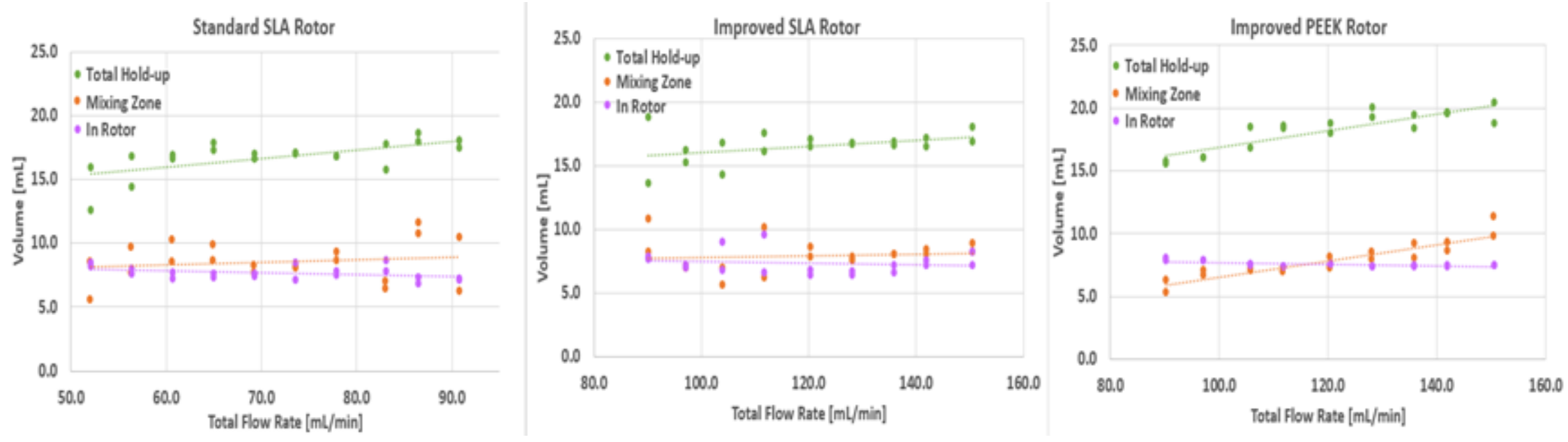

FIGURE 13 Holdup volume $[\mathrm{mL}]$ vs total feed rate $[\mathrm{mL} / \mathrm{min}]$.

N
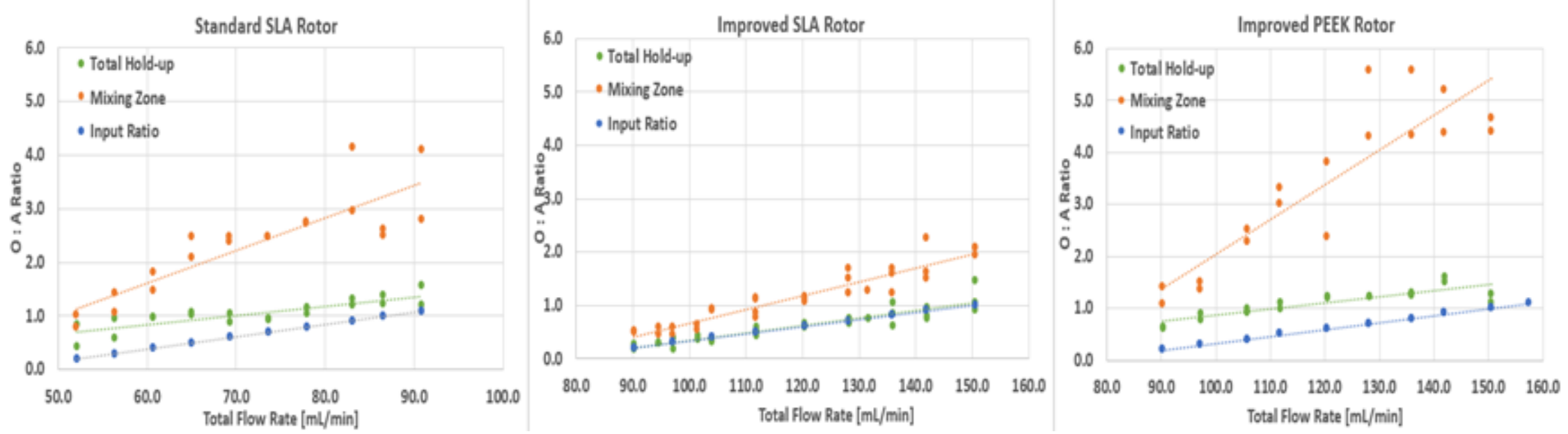

FIGURE 14 O:A ratio of holdup vs total flow rate $[\mathrm{mL} / \mathrm{min}] .^{5}$

${ }^{5}$ Candido Pereira, personal communication, October 10, 2019. 


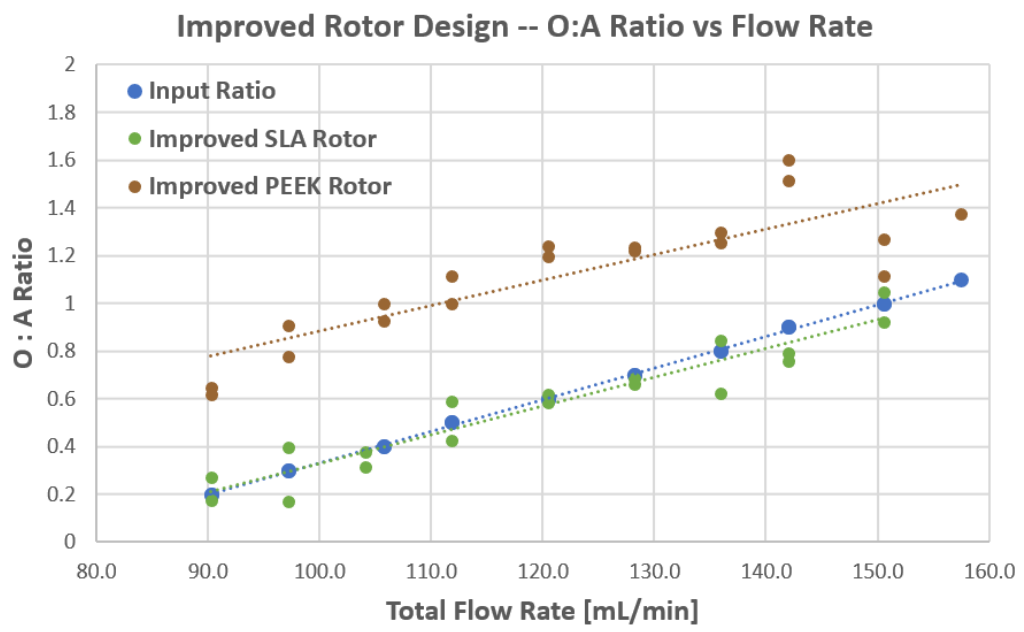

FIGURE 15 O:A ratios of the holdup volume within $2 \mathrm{~cm}$ stages for various flow rates [mL/min]. 
This page intentionally left blank. 



\section{Argonne $\mathbf{A}$}

\section{Chemical \& Fuel Cycle Technologies Division}

Argonne National Laboratory

9700 South Cass Avenue, Bldg. 205

Argonne, IL 60439-4832

www.anl.gov 